\title{
CLOSED OPERATORS AND PURE CONTRACTIONS IN HILBERT SPACE ${ }^{1}$
}

\author{
WILLIAM E. KAUFMAN
}

\begin{abstract}
Some properties of the one-to-one mapping $A \rightarrow A\left(1-A^{*} A\right)^{-1} 2$ of the pure contractions onto the closed and densely-defined operators are proved, in particular that it commutes with adjunction and preserves normality.
\end{abstract}

In a Hilbert space $H$, a pure contraction is a linear operator $A$ on $H$ such that $\|A x\|<\|x\|$ for all $x \neq 0$ in $H$. In [2] I showed that the function $\Gamma$ defined by $\Gamma(A)=A\left(1-A^{*} A\right)^{-1 / 2}$ maps the set of all pure contractions one-to-one onto the set of all closed and densely-defined operators in $H$. Since $\Gamma$ preserves many properties of operators, it may be used to reformulate questions about unbounded operators in terms of bounded ones. For example, in [1, §5, pp. 708-712], Cordes and Labrousse prove that if a closed and densely-defined operator $C$ is semiFredholm then so is the bounded operator $C\left(1+C^{*} C\right)^{-1 / 2}$; the latter is simply $\Gamma^{-1}(C)$.

The purpose of the present report is to indicate some further properties of $\Gamma$, and to establish some connections between unbounded symmetric operators and certain hyponormal contractions, leading to a reduction of the problem of finding selfadjoint extensions of symmetric operators to a corresponding problem involving only bounded operators.

From this point on, $A$ denotes a pure contraction, $B$ and $B_{*}$ the associated defect operators $\left(1-A^{*} A\right)^{1 / 2}$ and $\left(1-A A^{*}\right)^{1 / 2}$, respectively, and $C$ the closed and densely-defined operator $\Gamma(A)=A B^{-1}$. (Note that $\Gamma\left(A^{*}\right)=A^{*} B_{*}^{-1}$.) We take for granted the following relations proved in [2]: $\operatorname{ran} B=\operatorname{dom} C, C^{*}=B^{-1} A^{*}, B=$ $\left(1+C^{*} C\right)^{-1 / 2}$, and (thus) $C^{*} C=B^{-2}-1$. Note also that since $A$ is a pure contraction, $B$ and $B^{*}$ are one-to-one.

THEOREM 1. The operator $C$ has an everywhere-defined and bounded inverse if and only if the operator $A$ is invertible.

Proof. Since $B$ is one-to-one and $\operatorname{ran} A=\operatorname{ran} C, A$ is invertible if and only if $C$ is one-to-one with range $H$. By the closed graph theorem, this is equivalent to $C$ having a bounded inverse with domain $H$.

LEMMA 1. $\Gamma$ commutes with adjunction, i.e., $\Gamma\left(A^{*}\right)=\Gamma(A)^{*}$.

Received by the editors September 16, 1981 and, in revised form, February 17, 1982.

1980 Mathematics Subject Classification. Primary 47A45, 47A65.

'This work was supported in part by an Ohio University Research Award. 
Proof. It is well known that $A^{*} B_{*}=B A^{*}$ and, dually, $A B=B_{*} A$ (e.g. see [3, pp. 6-7]). Hence

$$
\left.\Gamma(A)^{*}\right|_{\operatorname{ran} B_{*}}=B^{-1} A^{*} B_{*} B_{*}^{-1}=B^{-1} B A^{*} B_{*}^{-1}=\Gamma\left(A^{*}\right) .
$$

To complete the proof, we show that $\operatorname{dom} \Gamma(A)^{*} \subseteq \operatorname{ran} B_{*}$, i.e., if $A^{*} x$ is in $\operatorname{ran} B$ then $x$ is in $\operatorname{ran} B_{*}$. Let $A^{*} x=B y$; then $\left(1-B_{*}^{2}\right) x=A A^{*} x=A B y=B_{*} A y$ and thus $x=B_{*}\left(B_{*} x-A y\right)$, as was to be proved.

Lemma 2. If $A B=B A$ then $A B^{-1}=B^{-1} A$.

Proof. We modify the proof of Lemma 1: if $A B=B A$ then $B^{-1} A B B^{-1}=$ $B^{-1} B A B^{-1}$, so that $\left.B^{-1} A\right|_{\operatorname{ran} B}=A B^{-1}$. Let $A x=B y ;$ then

$$
\left(1-B^{2}\right) x=A^{*} A x=A^{*} B y=B A^{*} y
$$

(this last since $\left.A^{*} B=B A^{*}\right)$. Hence $x=B\left(B x-A^{*} y\right)$. Therefore $B^{-1} A=A B^{-1}$.

DEFINITION. The operator $C$ is quasinormal provided that $C\left(C^{*} C\right)=\left(C^{*} C\right) C$.

THEOREM 2. Both $\Gamma$ and $\Gamma^{-1}$ preserve normality and quasinormality. I.e., $C$ is normal (resp. quasinormal) if and only if $A$ is normal (resp. quasinormal).

Proof. We first observe that by Lemma 1 and the fact that $\Gamma$ is one-to-one, $\Gamma^{-1}\left(C^{*}\right)=A^{*}$ and thus $B_{*}=\left(1+C C^{*}\right)^{-1 / 2}$. From this, the equivalence of the normality of $A$ and $C$ may be seen as follows: $A$ is normal if and only if $\left(1-A^{*} A\right)^{1 / 2}=\left(1-A A^{*}\right)^{1 / 2}$ (i.e., $\left.B=B_{*}\right)$, which holds only in case $\left(1+C^{*} C\right)^{1 / 2}$ $=\left(1+C C^{*}\right)^{1 / 2}$, which in turn is true if and only if $C^{*} C=C C^{*}$.

Now suppose $A$ is quasinormal. Then $A B=B A$ and therefore by Lemma 2 , $C=A B^{-1}=B^{-1} A$. Hence (recall that $C^{*} C=B^{-2}-1$ ) we get that

$$
C\left(C^{*} C\right)=A B^{-1}\left(B^{-2}-1\right)=B^{-1} A B^{-2}-A B^{-1}=B^{-2} A B^{-1}-A B^{-1}=\left(C^{*} C\right) C .
$$

Conversely, $C$ quasinormal implies $C B=B C$ and therefore $A B=C B^{2}=B(C B)=$ $B A$. from which it follows at once that $A$ is quasinormal.

Lemma 3. $\Gamma(|A|)=|C|$, and if $V|A|$ is the polar decomposition of $A$ then $V|C|$ is the polar decomposition of $C$.

Proof. By definition, $|T|=\left(T^{*} T\right)^{1 / 2}$. We calculate

$$
|C|=\left(B^{-2}-1\right)^{1 / 2}=\left(1-B^{2}\right)^{1 / 2} B^{-1}=\left(A^{*} A\right)^{1 / 2} B^{-1}=\Gamma(|A|),
$$

and thus $V|C|=V|A| B^{-1}=A B^{-1}=C$.

Remarks. By Lemma 1, $\Gamma$ preserves selfadjointness (if $A=A^{*}$ then $C=\Gamma(A)=$ $\left.\Gamma\left(A^{*}\right)=\Gamma(A)^{*}=C^{*}\right)$; Lemma 3 may be viewed as stating that $\Gamma$ "preserves argument." With the usual identification of the complex numbers as scalar multiplications on $H, \Gamma^{-1}$ is seen to be an extension of the radial compression $z \rightarrow$ $z\left(1+|z|^{2}\right)^{-1 / 2}$ of the complex plane onto the unit circular disc. As we proved in [2], the "extended compression" $\Gamma^{-1}$ throws the bounded operators on $H$ onto the interior of the unit ball in the algebra of all bounded operators on $H$, whereas each unbounded closed and densely-defined operator is mapped to its boundary. Except for the factor $\sqrt{2}$, the unit circle in the plane is left invariant under $\Gamma^{-1}$. The following result characterizes the invariants of $\sqrt{2} \Gamma^{-1}$ : 
THEOREM 3. An operator is invariant under $\sqrt{2} \Gamma^{-1}$ if and only if it is a partial isometry.

Proof. Suppose $C$ is a partial isometry. Then $|C|$ is a projection, say $P$. Hence by Lemma 3, with $V$ as indicated therein,

$$
\begin{aligned}
A & =V \Gamma^{-1}(P)=V P(1+P)^{-1 / 2}=V P(1-P+2 P)^{-1 / 2} \\
& =V P(1-P)+V \sqrt{\frac{1}{2}} P=\sqrt{\frac{1}{2}} C .
\end{aligned}
$$

Conversely, suppose $\sqrt{2} A=C$. Then $B^{-1} C^{*}=B^{-1} \sqrt{2} A^{*}=\sqrt{2} C^{*} ;$ i.e., $B^{-1}=\sqrt{2}$ on $\operatorname{ran} C^{*}$. Hence for all $x$ in $\operatorname{ran} C^{*}$,

$$
\begin{aligned}
\|C x\|^{2}-\|x\|^{2} & =\|x\|^{2}+\|C x\|^{2}-2\|x\|^{2}=\left\|\left(1+C^{*} C\right)^{1 / 2} x\right\|^{2}-\|\sqrt{2} x\|^{2} \\
& =\left\|B^{-1} x\right\|^{2}-\|\sqrt{2} x\|^{2}=0 .
\end{aligned}
$$

This confirms that the restriction of $C$ to $\operatorname{ran} C^{*}$ is an isometry, which implies that $C$ is a partial isometry.

THeORem 4. $C$ is symmetric if and only if $A^{*} B$ is selfadjoint, in which case $A$ is hyponormal.

Proof. Let $\langle\cdot, \cdot\rangle$ denote the inner product in $H$. Then $C$ is symmetric if and only if, for all $(x, y)$ in $H \times H,\langle C B x, B y\rangle=\langle B x, C B y\rangle$; since $A=C B$, this is equivalent to $\left\langle x, A^{*} B y\right\rangle=\left\langle A^{*} B x, y\right\rangle$, which is simply the selfadjoint condition on $A^{*} B$. Thus $C$ is symmetric only in case $A^{*} B$ is selfadjoint. Now suppose that this is indeed the case: for all $x$ in $\operatorname{ran} B(=\operatorname{dom} C)$,

$$
\begin{aligned}
\left\|B_{*}^{-1} x\right\|^{2} & =\left\|\left(1+C C^{*}\right)^{1 / 2} x\right\|^{2}=\|x\|^{2}+\left\|C^{*} x\right\|^{2} \\
& =\|x\|^{2}+\|C x\|^{2}=\left\|B^{-1} x\right\|^{2} .
\end{aligned}
$$

This establishes that $B_{*}^{-1} B$ is an isometry on $H$ and therefore $B_{*}^{-1} B^{2} B_{*}^{-1} \subseteq$ $\left(B_{*}^{-1} B\right)\left(B_{*}^{-1} B\right)^{*} \leqslant 1$, so that $B^{2} \leqslant B_{*}^{2}$. Hence $A A^{*}=1-B_{*}^{2} \leqslant 1-B^{2}=A^{*} A$, which is to say that $A$ is hyponormal.

As a further example of applying facts about $\Gamma$ to questions concerning unbounded operators, there is the following:

THEOREM 5. If a closed and densely-defined quasinormal operator is symmetric then it is selfadjoint.

Proof. If $C$ is quasinormal then by Theorem $2, A=\Gamma^{-1}(C)$ is also. If $C$ is symmetric then by Theorem $4, A^{*} B=\left(A^{*} B\right)^{*}=B A$. But since $A$ is quasinormal, $B A=A B$; thus $A^{*}$ agrees with $A$ on the dense subspace $\operatorname{ran} B$ of $H$. Hence $A=A^{*}$ and thus by Lemma $1, C=C^{*}$.

For the remainder of this report, we focus on the relationship between $C$ and $A B_{*}$ when $C$ is symmetric. Note that in this case if $A B_{*}$ is selfadjoint then by Theorem 4 , $C^{*}$ is symmetric, so that $C$ is selfadjoint. We will show that the search for selfadjoint extensions of $C$ is reducible to the finding of maximal linear subspaces $M$ of $H$ on which the bounded operator $A B_{*}$ is symmetric, in the sense that for all $x$ and $y$ in $M$, $\left\langle A B_{*} x, y\right\rangle=\left\langle x, A B_{*} y\right\rangle$. We start with a technical lemma. 
LEMMA 4. If $C$ is symmetric then $A^{2}+B_{*} B$ is the isometry $B_{*}^{-1} B$ from $H$ onto $B_{*}^{-1}(\operatorname{dom} C)$, and $\left(A B_{*}-B_{*} A\right)\left(A^{2}+B_{*} B\right)=0$.

Proof. We recall from the proof of Theorem 4 the fact that $B_{*}^{-1} B$ is an isometry; clearly its range is $B_{*}^{-1}(\operatorname{dom} C)$. Using the relations $C^{*} B=C B=A(C$ is symmetric $)$ and $A A^{*}+B_{*}^{2}=1$, we get that

$$
B_{*}^{-1} B=A A^{*} B_{*}^{-1} B+B_{*}^{2} B_{*}^{-1} B=A C^{*} B+B_{*} B=A^{2}+B_{*} B
$$

The rest of the lemma is now available by direct calculation:

$$
\begin{aligned}
\left(A B_{*}-B_{*} A^{*}\right)\left(A^{2}+B_{*} B\right) & =\left(A B_{*}-B_{*} A^{*}\right) B_{*}^{-1} B=A B-B_{*} C^{*} B \\
& =A B-B_{*} C B=A B-B_{*} A=0 .
\end{aligned}
$$

THEOREM 6. If $C$ is symmetric then an operator $T$ is a symmetric extension of $C$ if and only if $T=\left.C^{*}\right|_{B_{*}(M)}$, where $M$ is a linear subspace of $H$ containing $\operatorname{ran}\left(A^{2}+B_{*} B\right)$ and on which $A B_{*}$ is symmetric. In this case, $T$ is closed if and only if $M$ is closed.

Proof. Note that the equation $\left(A B_{*}-B_{*} A^{*}\right)\left(A^{2}+B_{*} B\right)=0$ of Lemma 4 assures us that $A B_{*}$ is automatically symmetric on $\operatorname{ran}\left(A^{2}+B_{*} B\right)$. It is elementary that an operator $T$ is a symmetric extension of $C$ only in case $T=\left.C^{*}\right|_{S}$, where $S$ is a linear subspace of $\operatorname{dom} C^{*}$ including $\operatorname{dom} C$, and on which $C^{*}$ is symmetric. Now $\operatorname{dom} C^{*}=\operatorname{ran} B^{*}$ and, since $C \subseteq C^{*}$ (so that $\operatorname{ran} B \subseteq \operatorname{ran} B^{*}$ ), we know also that $\operatorname{dom} C=\operatorname{ran} B_{*} B_{*}^{-1} B=B_{*}\left(\operatorname{ran} B_{*}^{-1} B\right)$. By Lemma $4, B_{*}^{-1} B=A^{2}+B_{*} B$; hence $\operatorname{dom} C=B_{*}\left(\operatorname{ran}\left(A^{2}+B_{*} B\right)\right)$. From this it is clear that the subspace $M$ promised by the theorem is simply $B_{*}^{-1}(S)$, and $M$ includes $\operatorname{ran}\left(A^{2}+B_{*} B\right)$ because $S$ includes $\operatorname{dom} C$. All that remains is to verify that the symmetry of $C^{*}$ on $S=B_{*}(M)$ is equivalent to the symmetry of $A B_{*}$ on $M$, viz.: for all $(x, y)$ in $M \times M$, $\left\langle A B_{*} x, y\right\rangle=\left\langle x, A B_{*} y\right\rangle$ if and only if $\left\langle B_{*} x, C^{*}\left(B_{*} y\right)\right\rangle=\left\langle C^{*}\left(B_{*} x\right), B_{*} y\right\rangle$, since $A^{*}=C^{*} B_{*}$.

Suppose now that $T$ is the symmetric extension $\left.C^{*}\right|_{B_{*}(M)}$ of $C$ and consider: $M$ is closed if and only if $B_{*}(M)$ is closed with respect to the norm $\left\|B_{*}^{-1} \cdot\right\|$ on $\operatorname{ran} B_{*}$. Since for all $x$ in $\operatorname{dom} C^{*}=\operatorname{ran} B_{*}$, the norm in $H \times H$ of the member $\left(x, C^{*} x\right)$ of $C^{*}$ is given by $\left\|B_{*}^{-1} x\right\|=\left\|\left(1+C C^{*}\right)^{1 / 2} x\right\|=\left(\|x\|^{2}+\left\|C^{*} x\right\|^{2}\right)^{1 / 2}$, we conclude that $M$ is closed if and only if $T$ is closed, and the proof is complete.

REMARK. Since the equations $T=\left.C^{*}\right|_{B_{*}(M)}$ define an inclusion-preserving mapping from the symmetric extensions $T$ of $C$ onto certain subspaces $M$ of $H$, one might inquire as to whether there is available a natural adjunction for such subspaces $M$. This is indeed the case.

THEOREM 7. If $C$ is symmetric and $T=\left.C^{*}\right|_{B_{*}(M)}$ is a symmetric extension of $C$, then $T^{*}=\left.C^{*}\right|_{B_{*}\left(M^{*}\right)}$, where $M^{*}=\left(A B_{*}-B_{*} A^{*}\right)(M)^{\perp}$.

Proof. For all $x$ in $H, B_{*} x$ is in $\operatorname{dom} T^{*}$ if and only if for all $y$ in $M$, $\left\langle B_{*} x, C^{*} B_{*} y\right\rangle=\left\langle C^{*} B_{*} x, B_{*} y\right\rangle$; equivalently,

$$
\begin{aligned}
\left\langle x,\left(A B_{*}-B_{*} A^{*}\right) y\right\rangle & =\left\langle A^{*} x, B_{*} y\right\rangle-\left\langle B_{*} x, A^{*} y\right\rangle \\
& =\left\langle C^{*} B_{*} x, B_{*} y\right\rangle-\left\langle B_{*} x, C^{*} B_{*} y\right\rangle=0
\end{aligned}
$$


But the requirement that $\left\langle x,\left(A B_{*}-B_{*} A^{*}\right) y\right\rangle=0$ for all $y$ in $M$ is just the requirement that $x$ be in $\left(A B_{*}-B_{*} A^{*}\right)(M)^{\perp}=M^{*}$. Hence $\operatorname{dom} T^{*}=B_{*}\left(M^{*}\right)$, as was to be proved.

COROLlaRY. The extension $T$ is selfadjoint if and only if $M=M^{*}$, i.e., $M$ is a maximal subspace of $H$ on which $A B_{*}$ is symmetric.

ACKNOWLEDGEMENT. I am indebted to the referee for pointing out that the hyponormality of $A$ in Theorem 4 is a consequence of the fact that $B_{*}^{-1} B$ is an isometry.

\section{REFERENCES}

1. H. O. Cordes and J. P. Labrousse. The invariance of the index in the metric space of closed operators. J. Math. Mech. 12 (1963), 693-719.

2. W. E. Kaufman. Representing a closed operator as a quotient of continuous operators, Proc. Amer. Math. Soc. 72 (1978), 531-534.

3. B. Sz.-Nagy and C. Foias, Harmonic analysis of operators on Hilhert space. North-Holland. Amsterdam, 1970 .

Department of Mathematics. Ohio University. Athens. Ohio 45701 